\title{
Multihop Alternate Cluster Head Fault Tolerant Dynamic Clustering Protocol for Wireless Sensor Network
}

\author{
Rajvi Chinaiwala \\ M.E. Student, Computer \\ Sigma Institute of Engineering \\ Vadodara
}

\author{
Kishori Shekokar \\ H.O.D., Computer \\ Sigma Institute of Engineering \\ Vadodara
}

\begin{abstract}
This paper introduces MACH-FTDC (Multihop Alternate Cluster Head Fault Tolerant - Dynamic Clustering) protocol for wireless sensor network that provides fault tolerance as well as reduced data loss with mobility as compared to the existing FT-DSC protocol. It introduces mobility which is important to some of the real world applications. This protocol does tradeoff between sensor node energy and data availability. For most of the applications, data is of utmost importance and hence this protocol focuses more on data availability and reduces loss of data at the cost of energy. Wireless sensor network is prone to failure due many reasons such as hardware faults, damage to sensor nodes while its deployment, software faults, energy depletion etc. Due to this, wireless sensor network is required to be fault tolerant. MACH-FTDC has reduced data loss achieving more data availability and provides mobility to WSN which is necessary for some applications.
\end{abstract}

\section{Keywords}

Fault tolerance, Dynamic Clustering, FT-DSC, Mobility, MEFC.

\section{INTRODUCTION}

This Wireless Sensor Network (WSN) is the network of large number of sensor nodes having specific characteristics: low cost, low energy, and requires to be unattended, that sense the data and transmit it to the base station for further processing which is based on the application for which the network is designed. Each sensor node performs three functions: sensing, data processing and communication. Sensor nodes in WSN are deployed under the ruthless environment where it is required to work unattended. Sensor nodes have limited amount of energy so after sometime it might get void of energy. Also, some sensor nodes might get broken while their deployment due to this fact WSN are required to be fault tolerant. Routing is the biggest challenge in case of WSN due to its undefined topology and lack of infrastructure. Based on this fact, routing protocols for WSN are required to be dynamic and adaptive to the changes in topology of network area. Sensor nodes are prone to many kinds of faults due to which we require our routing protocols to be fault tolerant so that our network operations are performed undisrupted and data are sent by providing alternative paths. Amongst different types of routing protocols, hierarchical or clustering protocol is the most energy efficient.

As sensor nodes have limited bandwidth, energy, storage capacity and processing speed designing a fault tolerant and energy efficient clustering protocol that tradeoff between the energy and scalability is an important research issue in WSN [1]. Routing protocols for WSNs are very application specific. Different applications have different criteria and to fulfill that we have different routing protocols. After reviewing "Fault Tolerant Dynamic Clustering (FT-DSC) Protocol [1]", it has been found out that the FT-DSC protocol suffers from data loss. As what if the $\mathrm{CH}$ fails in between the round, the data sent between its failure time and from the beginning will be lost. Also, Detection of failed CH is delayed as BS has to wait until the completion of round when the $\mathrm{CH}$ has to send the data after aggregating them to the BS. Due to this, FT-DSC cannot be applied to the applications where data is of utmost importance. After reviewing "Mobility - Centric Energy Efficient and Fault Tolerant Clustering Protocol (MEFC) [2]", it has been found that similar to FT-DSC, it too suffers from data loss. What if Active Node (AN) fails and is unable to transmit data to the $\mathrm{CH}, \mathrm{CH}$ will have to wait for specific number of timeslots in order to detect that AN has failed. Till that time if the subscribed event occurs, the data will be lost. Thus, this paper proposes the mixture of both FT-DSC and MEFC, called Multihop Alternate Cluster Head Fault Tolerant Dynamic Clustering (MACH-FTDC).

This paper proposes a multihop alternate cluster head fault tolerant dynamic clustering protocol which overcomes the limitations of FT-DSC and also provides mobility as optional. In MACH-FTDC, $\mathrm{CH}$ aggregates and sends data periodically to the BS. In this protocol, tradeoff between data availability and energy is done. Also, the network setup in this protocol is same as MEFC (Mobility Centric Energy Efficient Fault tolerant Clustering) Protocol [2] instead of using network setup of FT-DSC.

The remainder of this paper is organized as follows. Section II has some important terminologies and assumptions made in this protocol. Section III has the proposed protocol MACHFTDC. Section IV has the simulation setup and its results. Section V has conclusion.

\section{PRELIMINARIES}

\subsection{Terminology}

Sensing Hole: It is a point or area in the network that is not sensed by any sensor node [2].

\subsection{Assumptions}

Following assumptions are made in proposed MACH-FTDC protocol [2]:

- Sensors are homogeneous in terms of initial energy.

- BS is assumed at the center.

- Node A is one-hop count away from node B if A can communicate with B.

- Sensor nodes have same sensing and communication range.

- Sensors are mobile. They are attached to the objects which are mobile.

Sensor nodes are similar in terms of mobility. 


\section{PROPOSED MACH-FTDC PROTOCOL}

\subsection{Network Setup Phase}

This phase is divided into two phases: Hop Count and Euclidean Distance Calculation and Selection Phase

1) HOP COUNT AND EUCLIDEAN DISTANCE CALCULATION: Initially, sensor nodes transmit their location and energy information to the BS through other nodes for centralized control [2]. Nodes i that are neighbour to the BS will have HopCount $=1$. These nodes will then send their coordinates to the BS which in turn calculates the Euclidean distance (EuclidDist) and transmits it back to the respective nodes.

Similarly, other nodes $\mathrm{j}$ that are neighboring nodes to nodes $\mathrm{i}$ will calculate HopCount and EuclidDist using following formula:

HopCount $[\mathrm{j}]=$ HopCount $[\mathrm{i}]+1$

EuclidDist $[\mathrm{j}]=$ EuclidDist $[\mathrm{i}, \mathrm{j}]+\operatorname{Dist}[\mathrm{i}, \mathrm{j}]$

2) SELECTION PHASE: In this phase, $\mathrm{CH}, \mathrm{ACH}, \mathrm{MN}, \mathrm{AN}$ and GW are selected.

Step 1: BS first selects the nodes that are one-HopCount away from $\mathrm{BS}$ as $\mathrm{CH}$. While doing this, if two neighbouring nodes (within the communication range of each other) have HopCount $=1$, BS will consider the node with least EuclidDist as $\mathrm{CH}$ and the other node will be considered as $\mathrm{ACH}$. Nodes which are one-HopCount away from the $\mathrm{CH}$ will join the cluster of the closest $\mathrm{CH}$ as $\mathrm{MN}$.

Step 2: Other nodes that are not selected as $\mathrm{MN}$ or $\mathrm{CH}$ or ACH will participate in this step. Node with lowest HopCount will be selected as $\mathrm{CH}$. Again if two neighbouring nodes a and $\mathrm{b}$ has same HopCount value, then the node with lowest EuclidDist will be selected as $\mathrm{CH}$ and other node will be selected as $\mathrm{ACH}$. Nodes that are one HopCount away from the selected $\mathrm{CH}$ will join the cluster of the closest $\mathrm{CH}$ to become MN. This procedure will be repeated until all the nodes become either $\mathrm{CH}$ or $\mathrm{ACH}$ or $\mathrm{MN}$.

In case of introducing mobility to the protocol: let $n 1, n 2, n 3$ $\ldots . \mathrm{nh}$ be the number of nodes at the HopCount 1, 2, $3 \ldots \mathrm{h}$ away from the BS. Total number of nodes $=\mathrm{N}$. To overcome the inefficiency that arises due to the disjoint sets or overlapping of all nodes (in terms of communication range), mobility is introduced. BS transmits control signals to the attached objects so that the nodes at one HopCount away from BS move, whenever all or none of them overlap in terms of communication range. This will have maximum number of nodes that are one-HopCount away and become $\mathrm{CHs}$ as $\left\lfloor\frac{\mathrm{n}_{1}}{2}\right\rfloor$

Step 3: After the clusters are formed, $\mathrm{CH}$ selects some $\mathrm{MN}$ as AN. These AN will sense and transmit data according to the TDMA scheme and all other MN will go in sleep mode. AN will be selected such that it reduces sensing hole as reduced transmission of redundant data.

Step 4: GW is selected to facilitate the inter-cluster communication and minimum number of GW selected is 1 . $\mathrm{GW}$ is selected such that it is within the communication range of two or more $\mathrm{CHs}$. If there are more than one such node that satisfies this criteria, pool of GW is formed with the priority set according to the minimum EuclidDist. A new GW is selected by $\mathrm{CH}$ after certain number of rounds if the remaining energy of the current GW goes below some threshold value to achieve longer network lifetime [2].

\subsection{Steady Phase}

BS initially subscribes all nodes for some event of interest based on the application but after that $\mathrm{CH}$ will subscribe the event to all the AN. All $\mathrm{CH}$ assigns the timeslots to their respective AN using the TDMA scheme to transmit the data sensed. AN will send either the sensed data or the small sized special packet (in case if the subscribed event does not occur) to the $\mathrm{CH}$ in its allocated time slot. If $\mathrm{CH}$ does not receives any packets from the AN in their timeslot, it will assume that AN to be failed, select new AN, and assign the timeslot of failed AN to the new AN. This provides fault tolerance for the AN.

$\mathrm{CH}$ aggregates the data received from ANs and transmit it to the BS periodically. If the BS does not receive any data packet from the $\mathrm{CH}$ periodically, it will send "Hello" packet to the $\mathrm{CH}$ and turn the timer on. If BS does not receive any "ACK" packet from the $\mathrm{CH}$, it will assume the $\mathrm{CH}$ to be failed. BS then selects $\mathrm{ACH}$ as the new $\mathrm{CH}$. This provides fault tolerance for the $\mathrm{CH}$.

\subsection{Mobility Management}

Mobility management of MACH-FTDC is similar to that of MEFC [2]. It supports mobility of sensor nodes in two ways:

1. Applications in which network coverage is of utmost importance: In this, BS sends messages to the nodes to move to an area A which is uncovered if it is possible for that node or make the awaken node in an area A as AN whenever an AN moves leaving the area A uncovered.

2. Applications where data availability is of utmost importance compared to the network coverage: All the nodes in this case will act as AN. In case if some AN, x moves out of the cluster, it will send "JOIN CLUSTER REQUEST" to the closest $\mathrm{CH}, \mathrm{y}$. If $\mathrm{y}$ has an empty slot for $\mathrm{AN}, \mathrm{x}$, it will assign the timeslot to $\mathrm{x}$ after sending "ACCEPT JOIN" message to $x$

\section{SIMULATION SETUP \& RESULTS}

We have simulated the proposed MACH-FTDC in ns2. The following table shows the main network parameters considered in the simulations:

Table 1. Simulation parameters and their values

\begin{tabular}{|c|c|}
\hline Parameters & Value \\
\hline Network Size & $500 \times 500 \mathrm{~m}^{2}$ \\
\hline Maximum number of nodes & 150 \\
\hline Number of clusters & $4-12$ \\
\hline BS position & $250 \times 250 \mathrm{~m}$ \\
\hline MAC & 802.11 \\
\hline Initial Node Energy & $2 \mathrm{~J}$ \\
\hline
\end{tabular}

The simulation was carried out with initial energy of $2 \mathrm{~J}$ for each node and total number of node $=100$. Energy model used in simulation of MACH - FTDC is the default energy model of ns2. Following is the graph that compares both FTDSC and MACH - FTDC (with and without mobility). It shows that energy consumed in FT-DSC is less compared to 
MACH - FTDC as the residual energy of the WSN in MACH - FTDC is more compared to FT-DSC. Inspite of having being done trade - off between data availability and energy, the residual energy of MACH - FTDC is more as compared to FT-DSC because of its network setup. The network setup in MACH-FTDC is not frequently done due to which lot of energy is saved.

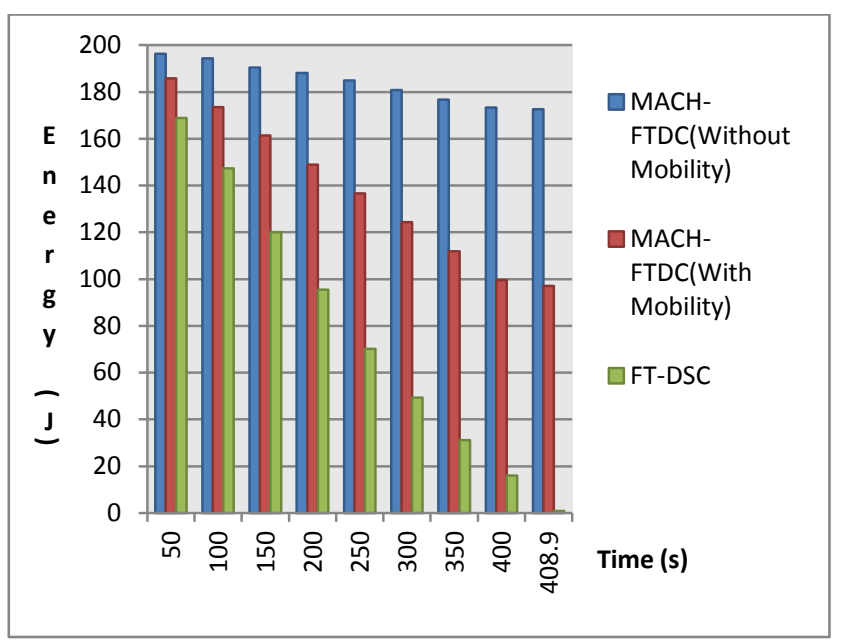

Fig 1: Comparison of Residual Energy

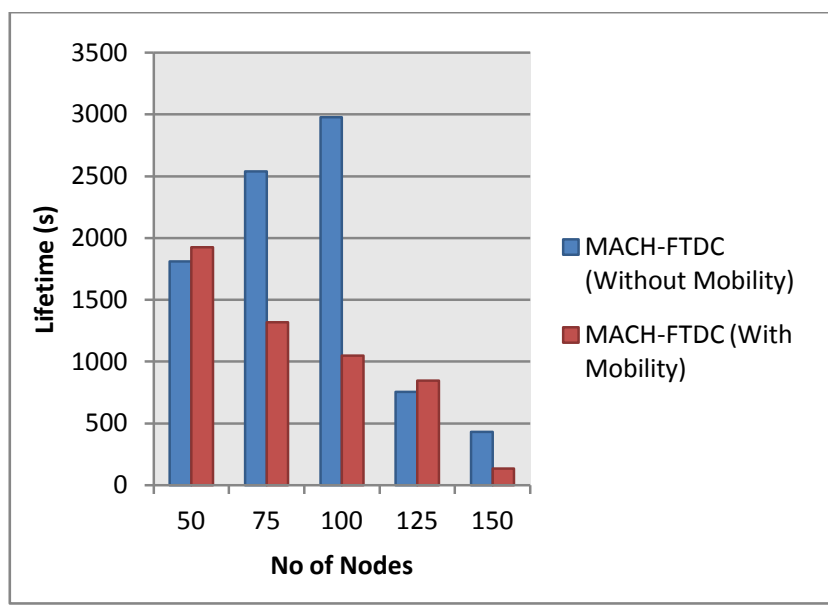

Fig 2: No of nodes vs. Lifetime.
Figure 3 shows scalability result of MACH - FTDC (with and without mobility). This graph shows that MACH-FTDC (with and without Mobility) is scalable up to some extent.

\section{CONCLUSION \& FUTURE WORK}

Hereby it is concluded that our proposed algorithm supports fault tolerance as well as mobility to the nodes. It improves the data availability as compared to the FT-DSC by doing trade - off between data availability and energy.

As a future work, we are planning to implement this protocol over different MACs and test the results for this protocol.

\section{REFERENCES}

[1] Lutful Karim, Nidal Nasser, Tarek Sheltami, "A Fault Tolerant Dynamic Clustering Protocol of Wireless Sensor Networks", In Global Telecommunications Conference, 2009. GLOBECOM 2009, IEEE, Nov. 30 2009-Dec. 4 2009, ISSN: 1930 - 529X

[2] Lutful Karim, Jalal Almhana and Nidal Nasser, "Mobility - Centric Energy Efficient and Fault Tolerant Clustering Protocol of Wireless Sensor Network", In IEEE ICC Ad-hoc and Sensor Networking Symposium, 2013, 9-13 June 2013, ISSN: 1550-3607, pp. 1479 1484.

[3] Lutful Karim, Nidal Nasser and Tarek Sheltami, "A fault-tolerant energy-efficient clustering protocol of a wireless sensor network", In Wiley Online Library, 26 January 2012, wireless communications and mobile computing, pp. 175-185

[4] Fuad Bajaber, Irfan Awan, "Dynamic/Static Clustering Protocol for Wireless Sensor Network", In Computer Modeling and Simulation, 2008, EMS '08. Second UKSIM European Symposium on 8-10 Sept. 2008, EISBN: 978-0-7695-3325-4, pp. 524-529.

[5] Ms. Rajvi K. Chinaiwala and Ms. Kishori Shekokar, "A Brief Survey on Clustering Routing Protocols for Wireless Sensor Networks." International Journal for Scientific Research and Development 2.10 (2014): 136140.

[6] Tripti Singh, Neha Gupta, Jasmine Minj, "Hierarchical Cluster Based Routing Protocol with High Throughput for Wireless Sensor Networks", In Signal Processing, Computing and Control (ISPCC), 2013 IEEE International Conference on 26-28 Sept. 2013, pp 1-6, Print ISBN: 978-1-4673-6188-0. 